Modelling, Analysis and Simulation

MAS $\begin{aligned} & \text { Modelling, Analysis and Simulation } \\ & \text { Conservation of wave action under multisymplectic } \\ & \text { discretizations }\end{aligned}$

J.E. Frank

REPORT MAS-R0602 JANUARY 2006 
Centrum voor Wiskunde en Informatica (CWI) is the national research institute for Mathematics and Computer Science. It is sponsored by the Netherlands Organisation for Scientific Research (NWO).

CWI is a founding member of ERCIM, the European Research Consortium for Informatics and Mathematics.

CWI's research has a theme-oriented structure and is grouped into four clusters. Listed below are the names of the clusters and in parentheses their acronyms.

Probability, Networks and Algorithms (PNA)

Software Engineering (SEN)

\section{Modelling, Analysis and Simulation (MAS)}

Information Systems (INS)

Copyright (C) 2006, Stichting Centrum voor Wiskunde en Informatica

P.O. Box 94079, 1090 GB Amsterdam (NL)

Kruislaan 413, 1098 SJ Amsterdam (NL)

Telephone +31205929333

Telefax +31205924199

ISSN 1386-3703 


\title{
Conservation of wave action under multisymplectic discretizations
}

\begin{abstract}
In this paper we discuss the conservation of wave action under numerical discretization by variational and multisymplectic methods. Both the general wave action conservation defined with respect to a smooth, periodic, one-parameter ensemble of flow realizations and the specific wave action based on an approximated and averaged Lagrangian are addressed in the numerical context. It is found that the discrete variational formulation gives rise in a natural way not only to the discrete wave action conservation law but to a generalization of the numerical dispersion relation to the case of variable coefficients. Indeed a fully discrete analog of the modulation equations arises. On the other hand the multisymplectic framework gives easy access to the conservation law for the general class of multisymplectic Runge-Kutta methods. A numerical experiment confirms conservation of wave action to machine precision and suggests that the solution of the discrete modulation equations approximates the numerical solution to order $\mathcal{O}(\varepsilon)$ on intervals of $\mathcal{O}\left(\varepsilon^{-1}\right)$.
\end{abstract}

2000 Mathematics Subject Classification: 65P10,74S10,74J30,76B15

Keywords and Phrases: numerical discretization of PDEs; wave equations; wave action; discrete variational integrators; multisymplectic integrators

Note: Work carried out under Project MAS 1.3, 'Numerical modelling of atmosphere and ocean'. Funding through a Veni grant from the Netherlands Organization for Scientific Research is gratefully acknowledged. 



\title{
Conservation of wave action under multisymplectic discretizations
}

\author{
Jason Frank \\ CWI, P.O. Box 94079, 1090 GB Amsterdam, The Netherlands \\ E-mail: jason@cwi.nl
}

\begin{abstract}
In this paper we discuss the conservation of wave action under numerical discretization by variational and multisymplectic methods. Both the general wave action conservation defined with respect to a smooth, periodic, oneparameter ensemble of flow realizations and the specific wave action based on an approximated and averaged Lagrangian are addressed in the numerical context. It is found that the discrete variational formulation gives rise in a natural way not only to the discrete wave action conservation law but to a generalization of the numerical dispersion relation to the case of variable coefficients. Indeed a fully discrete analog of the modulation equations arises. On the other hand the multisymplectic framework gives easy access to the conservation law for the general class of multisymplectic Runge-Kutta methods. A numerical experiment confirms conservation of wave action to machine precision and suggests that the solution of the discrete modulation equations approximates the numerical solution to order $\mathcal{O}(\varepsilon)$ on intervals of $\mathcal{O}\left(\varepsilon^{-1}\right)$.
\end{abstract}

AMS classification scheme numbers: 65P10,74S10,74J30,76B15

Submitted to: J. Phys. A: Math. Gen.

\section{Introduction}

The wave action conservation law was introduced in (Whitham 1965) to study modulations of wave trains in slowly varying media. The approach follows by substituting a wave train with slowly varying amplitude, wave number and frequency into the variational principle, neglecting terms of higher order in a small perturbation parameter, and averaging the Lagrangian over phase to arrive at a variational principle for the modulation equations. Whitham's original theory thus leads to an approximate conservation law. A generalized form of the conservation law of wave action was introduced by (Hayes 1970), who considered a periodic, one-parameter family of solutions to the Euler-Lagrange equations. Conservation of wave action then follows from Noether's theorem, due to the trivial invariance of the action integral under translations in the ensemble parameter. This in turn makes Hayes's theory an exact one. The identification of Hayes's ensemble parameter with a phase shift relates the two theories (see (Hayes 1970) and (Grimshaw 1984) for more discussion). The concept of wave action conservation was further extended in (Whitham 1970), and a full treatment is given in the monograph (Whitham 1999). 
The utility of the wave action conservation law is that it holds even when the action integral is explicitly dependent on time and space, such that the energymomentum tensor is not exactly conserved. An important example is the case of waves defined on a slowly moving background flow, such as shallow water gravity waves on a slowly evolving potential vorticity field. The theory has therefore found application in wave-mean field interactions (Bretherton \& Garrett 1969, Andrews \& McIntyre 1978, Grimshaw 1984). Another important application is the instability theory of traveling waves (see (Bridges 1997b, Bridges 1997a) and the references therein). The local conservation law for wave action is a space-time generalization of the concept of an adiabatic invariant in a classical mechanical system with slow dependence of the Hamiltonian on time (Arnold 1989).

In this paper we consider wave equations in one space and one time dimension with a single wave action ensemble parameter. The results are easily generalized to higher dimensional space-time, however (Frank 2006). In $\S 3$ we will show that a number of multisymplectic numerical discretizations as developed in (Bridges \& Reich 2001, Reich 2000b) satisfy a discrete conservation law of wave action in the sense of (Hayes 1970). This result is a corollary to the fact that multisymplectic semi-discretizations satisfy a semi-discrete energy-momentum conservation in each continuous (i.e. undiscretized) coordinate, which follows from the Noether theory for multisymplectic PDEs (Bridges 1997b). The latter has been shown for special cases in the literature (Reich 2000b), and a very general statement for the class of multisymplectic Runge-Kutta box schemes is treated in (Frank 2006). In $\S 4$ we prove wave action conservation for this class of methods in the current setting. Additionally we will consider in $§ 3.1 .1$ a discrete variational integrator (Marsden et al. 1998, Marsden \& West 2001) applied to the linear Klein-Gordon equation with slowly varying coefficients, for which a discrete averaged Lagrangian is obtained which yields discrete versions of the modulation equations. It is curious that the wave action conservation law so obtained is identical to the exact one obtained by ensemble averaging, i.e. no approximation is necessary in the discrete case. In other words Whitham's and Hayes's wave action concepts are equivalent in the discrete setting.

In $\S 2$ wave action conservation in the continuous case is reviewed, both in the Lagrangian and multisymplectic Hamiltonian settings. In $\S 5$ we conclude with a numerical computation of the linear Klein-Gordon equation with slowly varying coefficients.

To the best of our knowledge this is the first paper to study discrete conservation of wave action under numerical discretization. At this time, it is difficult to anticipate the significance of this property. One can say, however, that conservation of wave action is a property of certain solutions of continuous wave equations which is inherited under discretization by the variational and multisymplectic methods considered here, and as such it further attests to the realism of simulations by such methods.

\section{Review of wave action conservation for continuous systems}

\subsection{Wave action conservation over a continuous ensemble}

The concept of wave action conservation was developed in a variational setting. Consider a wave equation derivable from a variational principle with Lagrangian $L$ 
(Marsden \& Ratiu 1994):

$$
\mathcal{L}=\int L\left(u_{t}, u_{x}, u, t, x\right) d x d t
$$

The Euler-Lagrange equations are

$$
\frac{\delta \mathcal{L}}{\delta u}=\partial_{t}\left(\frac{\partial L}{\partial u_{t}}\right)+\partial_{x}\left(\frac{\partial L}{\partial u_{x}}\right)-\frac{\partial L}{\partial u}=0 .
$$

Due to the explicit dependence of $L$ on time $t$ and space $x$, solutions of the EulerLagrange equations will not conserve energy or momentum in general. Suppose, however, that (2) possesses an ensemble of solutions $u\left(t, x, \theta_{0}\right)$ that can be smoothly parameterized by a closed loop in phase space, with loop parameter $\theta_{0}$. The derivative of the Lagrangian with respect to this parameter is

$$
\frac{d L}{d \theta_{0}}=\frac{\partial L}{\partial u_{t}} u_{t \theta_{0}}+\frac{\partial L}{\partial u_{x}} u_{x \theta_{0}}+\frac{\partial L}{\partial u} u_{\theta_{0}}
$$

Solving (2) for $\frac{\partial L}{\partial u}$ and substituting into the above expression gives

$$
\frac{d L}{d \theta_{0}}=\partial_{t}\left(\frac{\partial L}{\partial u_{t}} u_{\theta_{0}}\right)+\partial_{x}\left(\frac{\partial L}{\partial u_{x}} u_{\theta_{0}}\right) .
$$

Integrating this relation around a loop in $\theta_{0}$ yields the conservation law of wave action:

$$
\partial_{t} \mathcal{A}+\partial_{x} \mathcal{B}=0, \quad \mathcal{A}=\frac{1}{2 \pi} \oint \frac{\partial L}{\partial u_{t}} u_{\theta_{0}} d \theta_{0}, \quad \mathcal{B}=\frac{1}{2 \pi} \oint \frac{\partial L}{\partial u_{x}} u_{\theta_{0}} d \theta_{0},(3)
$$

where $\mathcal{A}$ is the wave action density and $\mathcal{B}$ is the wave action flux.

2.1.1. Example: Klein-Gordon equation, averaged Lagrangian As a concrete example let us take the linear dispersive Klein-Gordon equation in a slowly varying medium:

$$
u_{t t}=\left(\alpha(t, x)^{2} u_{x}\right)_{x}-\beta(t, x)^{2} u .
$$

This equation was used in (Whitham 1970) to illustrate the above concepts. Equation (4) is the Euler-Lagrange equation associated to the action integral

$$
\mathcal{L}=\int \frac{u_{t}^{2}}{2}-\alpha(t, x)^{2} \frac{u_{x}^{2}}{2}-\beta(t, x)^{2} \frac{u^{2}}{2} d x d t .
$$

For this Lagrangian, the wave action density and flux (3) read

$$
\mathcal{A}=\frac{1}{2 \pi} \oint u_{t} u_{\theta_{0}} d \theta_{0}, \quad \mathcal{B}=\frac{1}{2 \pi} \oint \alpha^{2} u_{x} u_{\theta_{0}} d \theta_{0},
$$

In application of the theory to slow modulations of nearly periodic wave trains, we assume that $\alpha$ and $\beta$ are slowly varying with respect to time and space, i.e. $\alpha=\alpha(T, X), \beta=\beta(T, X)$, where $X=\varepsilon x, T=\varepsilon t$ for small parameter $\varepsilon$. We are interested in a family of nearly sinusoidal solutions with slowly varying amplitude, frequency, and wave number, parameterized by a phase shift. To that end we make the ansatz

$$
\begin{aligned}
& u(t, x)=A(T, X) \sin \left(\theta(t, x)+\theta_{0}\right), \\
& \theta(t, x)=\varepsilon^{-1} \Theta(T, X) \\
& \theta_{t}(t, x)=-\omega(T, X) \\
& \theta_{x}(t, x)=\kappa(T, X)
\end{aligned}
$$


where $\theta_{0}$ is a phase shift. For such a solution, the wave action density and flux (6) can be integrated to yield

$$
\mathcal{A}=-\frac{1}{2} A^{2} \omega, \quad \mathcal{B}=\frac{1}{2} \alpha^{2} A^{2} \kappa .
$$

An alternative derivation proceeds by substituting (7) directly into the action integral (5) and averaging over $\theta_{0}$. The averaged Lagrangian is

$$
\begin{aligned}
\bar{L} & =\frac{1}{2 \pi} \oint L\left(u_{t}, u_{x}, u, t, x\right) d \theta_{0} \\
& =\frac{1}{2}\left[\frac{A^{2} \theta_{t}^{2}}{2}+\varepsilon^{2} \frac{A_{T}^{2}}{2}\right]-\frac{1}{2} \alpha^{2}\left[\frac{A^{2} \theta_{x}^{2}}{2}+\varepsilon^{2} \frac{A_{X}^{2}}{2}\right]-\frac{1}{2} \beta^{2} \frac{A^{2}}{2}
\end{aligned}
$$

Neglecting terms of order $\varepsilon^{2}$ gives the action integral

$$
\overline{\mathcal{L}}=\int \frac{1}{4}\left[A^{2} \theta_{t}^{2}-\alpha^{2} A^{2} \theta_{x}^{2}-\beta^{2} A^{2}\right] d x d t
$$

in terms of $A$ and $\theta$. The Euler-Lagrange equations for this action principle are

$$
\begin{aligned}
& \frac{\delta \overline{\mathcal{L}}}{\delta \theta}=\partial_{t}\left(\frac{1}{2} A^{2} \theta_{t}\right)+\partial_{x}\left(\frac{1}{2} \alpha^{2} A^{2} \theta_{x}\right)=0, \\
& \frac{\delta \overline{\mathcal{L}}}{\delta A}=\theta_{t}^{2}-\alpha^{2} \theta_{x}^{2}-\beta^{2}=0 .
\end{aligned}
$$

By making use of (8) and (9), one can express the above two equations in terms of the slowly varying quantities $\omega, \kappa$ and $A$ :

$$
\begin{aligned}
& \partial_{t}\left(-\frac{1}{2} A^{2} \omega\right)+\partial_{x}\left(\frac{1}{2} \alpha^{2} A^{2} \kappa\right)=0, \\
& \omega^{2}-\alpha^{2} \kappa^{2}-\beta^{2}=0, \\
& \kappa_{t}+\omega_{x}=0,
\end{aligned}
$$

where the last of these is the compatibility condition $\theta_{x t}=\theta_{t x}$.

Equations (13)-(15) are the modulation equations which (approximately) govern the evolution of the envelope of the slowly varying wave train. The first of these is just the wave action conservation law (3) for the specific case (10). Equation (14) is a generalization of the dispersion relation to the case of variable coefficients.

It has been noted in (Whitham 1970) that although (3), (10) were obtained directly through an ensemble average over $\theta_{0},(13)$ was only obtained after neglecting terms of higher order in $\varepsilon$, and is therefore an approximate conservation law. We will refer to the former, exact conservation with respect to an ensemble of flow realizations as Hayes's wave action, and the latter, approximate conservation law as Whitham's wave action. This paper primarily deals with the former, although for specific examples we will always turn to the latter.

Note that, while (13) is derived by considering a family of solutions, in its final form it applies to the amplitude, frequency and wave number of an individual solution out of that family (it is local in the ensemble variable). This conservation law holds even when the Lagrangian (1) depends explicitly on $t$ and $x$, i.e. when energy and momentum are not conserved. 


\subsection{Multisymplectic structure and wave action}

By taking a complete Legendre transformation of (1) not only with respect to $u_{t}$ but also with respect to $u_{x}$, one may derive a Hamiltonian wave equation in the abstract multisymplectic form (Bridges 1997b)

$$
J \boldsymbol{u}_{t}+K \boldsymbol{u}_{x}=\nabla S(\boldsymbol{u}, t, x),
$$

where $\boldsymbol{u}(t, x) \in \boldsymbol{R}^{N}, J^{T}=-J$ and $K^{T}=-K$ are $N \times N$ skew-symmetric matrices, and $S: \boldsymbol{R}^{N} \times \boldsymbol{R} \times \boldsymbol{R} \rightarrow \boldsymbol{R}$ is a functional which may depend on $t$ and $x$. The papers (Bridges 1997a) and (Bridges 1997b) provide a complete and accessible introduction to multisymplectic structure and some of its applications.

Suppose $S$ has no explicit dependence on $t$ in (16). Then taking the vector inner product of (16) with $\boldsymbol{u}_{t}$ gives

$$
\boldsymbol{u}_{t}^{T} J \boldsymbol{u}_{t}+\boldsymbol{u}_{t}^{T} K \boldsymbol{u}_{x}=\boldsymbol{u}_{t}^{T} \nabla S(\boldsymbol{u}, x)
$$

The first term is zero by skew-symmetry of $J$. Using the identity

$$
\boldsymbol{u}_{t}^{T} K \boldsymbol{u}_{x}=\partial_{t}\left(\frac{1}{2} \boldsymbol{u}^{T} K \boldsymbol{u}_{x}\right)+\partial_{x}\left(\frac{1}{2} \boldsymbol{u}_{t}^{T} K \boldsymbol{u}\right)
$$

and the fact that the right side of (17) is just the total derivative of $S$ with respect to $t$, the conservation law

$$
e_{t}+f_{x}=0, \quad e=\frac{1}{2} \boldsymbol{u}^{T} K \boldsymbol{u}_{x}-S, \quad f=\frac{1}{2} \boldsymbol{u}_{t}^{T} K \boldsymbol{u}
$$

is obtained. In (Bridges 1997b) it is observed that this is the energy conservation law associated with the invariance of (16) to time translations. If $S$ does not depend explicitly on $x$, the associated momentum conservation law follows analogously by taking the inner product of (16) with $\boldsymbol{u}_{x}$.

The wave action conservation principle of (Hayes 1970) has been cast in multisymplectic form in (Bridges 1997a). The idea is to consider a one-parameter ensemble of solutions $\boldsymbol{u}\left(t, x, \theta_{0}\right)$ to (16) smoothly parameterized by a closed loop in phase space $\theta_{0} \in \mathcal{S}^{1}$. Taking the vector inner product of (16) with $\boldsymbol{u}_{\theta_{0}}$ and using the same reasoning as above yields the conservation law

$\partial_{\theta_{0}}\left(\frac{1}{2} \boldsymbol{u}^{T} J \boldsymbol{u}_{t}+\frac{1}{2} \boldsymbol{u}^{T} K \boldsymbol{u}_{x}-S\right)+\partial_{t}\left(\frac{1}{2} \boldsymbol{u}_{\theta_{0}}^{T} J \boldsymbol{u}\right)+\partial_{x}\left(\frac{1}{2} \boldsymbol{u}_{\theta_{0}}^{T} K \boldsymbol{u}\right)=0$.

The ensemble average gives Hayes's conservation law of wave action

$\partial_{t} \mathcal{A}+\partial_{x} \mathcal{B}=0, \quad \mathcal{A}=\frac{1}{4 \pi} \oint \boldsymbol{u}_{\theta_{0}}^{T} J \boldsymbol{u} d \theta_{0}, \quad \mathcal{B}=\frac{1}{4 \pi} \oint \boldsymbol{u}_{\theta_{0}}^{T} K \boldsymbol{u} d \theta_{0}$

2.2.1. Example: multisymplectic description of the Klein-Gordon equation The Klein-Gordon equation can be cast in the form (16) by introducing the Legendre transformations $v:=\partial L / \partial u_{t}=u_{t}$ and $w:=\partial L / \partial u_{x}=-\alpha^{2} u_{x}$. Then one finds, with $\boldsymbol{u}=(u, v, w)$, that $S=\frac{1}{2}\left(v^{2}-\alpha^{-2} w^{2}+\beta^{2} u^{2}\right)$ and

$$
J=\left(\begin{array}{ccc}
0 & -1 & 0 \\
1 & 0 & 0 \\
0 & 0 & 0
\end{array}\right), \quad K=\left(\begin{array}{ccc}
0 & 0 & -1 \\
0 & 0 & 0 \\
1 & 0 & 0
\end{array}\right)
$$

The conservation law (21) holds with

$$
\mathcal{A}=\frac{1}{4 \pi} \oint v_{\theta_{0}} u-u_{\theta_{0}} v d \theta_{0}=-\frac{1}{2 \pi} \oint u_{\theta_{0}} u_{t} d \theta_{0}
$$


and

$$
\mathcal{B}=\frac{1}{4 \pi} \oint w_{\theta_{0}} u-u_{\theta_{0}} w d \theta_{0}=\frac{1}{2 \pi} \oint \alpha^{2} u_{\theta_{0}} u_{x} d \theta_{0}
$$

which are precisely (6).

The extension of this theory to higher dimensional space-time is straightforward. Additional dimensions may be included within the multisymplectic framework with an additional term $K^{(d)} \boldsymbol{u}_{x_{d}}\left(K^{(d)}\right.$ skew-symmetric) for each additional coordinate $x_{d}$. If $S$ is independent of $x_{d}$, then a momentum equation analogous to (19) may be found by taking the inner product of (16) with $\boldsymbol{u}_{x_{d}}$ and applying the identity (18). Indeed, the wave action conservation law (20) can also be derived in this way by considering $x_{0} \equiv \theta_{0}$ as an additional spatial dimension with periodic boundary conditions, with associated trivial skew-symmetric matrix $K^{0}=0$. Then it is the translation symmetry in $\theta_{0}$ which leads to (20). For a general statement in the context of multisymplectic Runge-Kutta discretizations, see (Frank 2006).

In (Bridges 1997a) it is shown that (21) is equivalent via Stokes theorem to a local conservation law of symplecticity. The defining property of a multisymplectic numerical discretization is that it satisfies a discrete version of the local conservation law of symplecticity. However, since Stokes theorem does not in general hold after discretization, it is not immediate that multisymplectic discretizations have an analogous wave action conservation law. In the next section we identify such conservation laws for some discrete variational and multisymplectic methods.

\section{Discrete wave action conservation}

\subsection{Wave action conservation for a discrete variational integrator}

Below we follow a derivation analogous to that of $\$ 2.1$ for a discrete variational integrator, see (Marsden et al. 1998, Marsden \& West 2001). Define a discrete Lagrangian by

$$
L_{i}^{n}:=L\left(\frac{u_{i}^{n+1}-u_{i}^{n}}{\Delta t}, \frac{u_{i+1}^{n}-u_{i}^{n}}{\Delta x}, u_{i}^{n}, x_{i}, t_{n}\right) .
$$

The discrete action integral (up to boundary conditions) is

$$
\mathcal{L}_{D}=\sum_{i, n} L_{i}^{n}
$$

and the discrete Euler-Lagrange equations are given by

$$
0=\frac{\partial \mathcal{L}_{D}}{\partial u_{i}^{n}}=\frac{1}{\Delta t}\left(L_{1 i}^{n}-L_{1 i}^{n-1}\right)+\frac{1}{\Delta x}\left(L_{2 i}^{n}-L_{2}^{n}{ }_{i-1}^{n}\right)-L_{3 i}^{n},
$$

where $L_{p_{i}}^{n}$ denotes the partial derivative of $L$ with respect to its $p$ th argument, evaluated at the same indices as (22). The scheme (23) will be referred to as the discrete variational Euler scheme.

Next we assume a family of discrete functions $u_{i}^{n}\left(\theta_{0}\right)$, satisfying (23) and smooth and periodic in $\theta_{0}$. We compute the derivative of $L_{i}^{n}$ with respect to $\theta_{0}$ :

$$
\frac{\partial L_{i}^{n}}{\partial \theta_{0}}=L_{1}^{n} \partial_{\theta_{0}} \frac{u_{i}^{n+1}-u_{i}^{n}}{\Delta t}+L_{2}^{n} \partial_{\theta_{0}} \frac{u_{i+1}^{n}-u_{i}^{n}}{\Delta x}+L_{3 i}^{n} \partial_{\theta_{0}} u_{i}^{n} .
$$

Substituting (23) into the last term on the right and rearranging gives

$\frac{\partial L_{i}^{n}}{\partial \theta_{0}}=\frac{1}{\Delta t}\left(L_{1 i}^{n} \partial_{\theta_{0}} u_{i}^{n+1}-L_{1}^{n-1} \partial_{\theta_{0}} u_{i}^{n}\right)+\frac{1}{\Delta x}\left(L_{2}^{n} \partial_{\theta_{0}} u_{i+1}^{n}-L_{2}^{n}{ }_{i-1} \partial_{\theta_{0}} u_{i}^{n}\right)$. 
Finally, taking the ensemble average yields a discrete conservation law. That is,

Proposition 1 The discrete variational Euler scheme (23) satisfies the discrete conservation law of wave action

$$
\frac{1}{\Delta t}\left(\mathcal{A}_{i}^{n+1 / 2}-\mathcal{A}_{i}^{n-1 / 2}\right)+\frac{1}{\Delta x}\left(\mathcal{B}_{i+1 / 2}^{n}-\mathcal{B}_{i-1 / 2}^{n}\right)=0
$$

where

$\mathcal{A}_{i}^{n+1 / 2}=\frac{1}{2 \pi} \oint L_{1 i}^{n} \partial_{\theta_{0}} u_{i}^{n+1} d \theta_{0}, \quad \mathcal{B}_{i+1 / 2}^{n}=\frac{1}{2 \pi} \oint L_{2 i}^{n} \partial_{\theta_{0}} u_{i+1}^{n} d \theta_{0}$.

Equation (24) is the discrete analog of (3).

3.1.1. Example: variational discretization of the Klein-Gordon equation Now let us consider a variational integrator for (4). The action principle (5) is approximated by the sum

$\mathcal{L}=\sum_{i, n} \frac{1}{2}\left(\frac{u_{i}^{n+1}-u_{i}^{n}}{\Delta t}\right)^{2}-\left(\alpha_{i+1 / 2}^{n}\right)^{2} \frac{1}{2}\left(\frac{u_{i+1}^{n}-u_{i}^{n}}{\Delta x}\right)^{2}-\left(\beta_{i}^{n}\right)^{2} \frac{1}{2}\left(u_{i}^{n}\right)^{2}$.

As in the continuous case, let us assume a family of discrete, slowly modulated waves of the form

$$
u_{i}^{n}=A_{i}^{n} \sin \left(\theta_{i}^{n}+\theta_{0}\right) .
$$

Substituting this family directly into (24) and taking the ensemble average yields the discrete wave action density

$$
\mathcal{A}_{i}^{n+1 / 2}=\frac{1}{2} A_{i}^{n} A_{i}^{n+1} \Delta t^{-1} \sin \left(\theta_{i}^{n+1}-\theta_{i}^{n}\right)
$$

and discrete flux

$$
\mathcal{B}_{i+1 / 2}^{n}=\frac{1}{2}\left(\alpha_{i+1 / 2}^{n}\right)^{2} A_{i}^{n} A_{i+1}^{n} \Delta x^{-1} \sin \left(\theta_{i+1}^{n}-\theta_{i}^{n}\right) .
$$

These quantities are second order approximations to (10).

It is also instructive to follow the averaged Lagrangian approach used in $\$ 2.1 .1$. Substituting (27) into (26) and averaging over $\theta_{0}$ gives the averaged variational principle

$$
\begin{aligned}
\overline{\mathcal{L}}=\sum_{i, n} & \frac{1}{4 \Delta t^{2}}\left[\left(A_{i}^{n+1}\right)^{2}-2 A_{i}^{n} A_{i}^{n+1} \cos \left(\theta_{i}^{n+1}-\theta_{i}^{n}\right)+\left(A_{i}^{n}\right)^{2}\right] \\
& +\frac{\left(\alpha_{i+1 / 2}^{n}\right)^{2}}{4 \Delta x^{2}}\left[\left(A_{i+1}^{n}\right)^{2}-2 A_{i}^{n} A_{i+1}^{n} \cos \left(\theta_{i+1}^{n}-\theta_{i}^{n}\right)+\left(A_{i}^{n}\right)^{2}\right] \\
& +\frac{\left(\beta_{i}^{n}\right)^{2}}{4}\left(A_{i}^{n}\right)^{2}
\end{aligned}
$$

Taking the discrete variation with respect to $\theta_{i}^{n}$ produces precisely (24) with (28) and (29):

$$
\begin{aligned}
0= & \frac{1}{2 \Delta t^{2}}\left[A_{i}^{n+1} A_{i}^{n} \sin \left(\theta_{i}^{n+1}-\theta_{i}^{n}\right)-A_{i}^{n} A_{i}^{n-1} \sin \left(\theta_{i}^{n}-\theta_{i}^{n-1}\right)\right]+ \\
& \frac{1}{2 \Delta x^{2}}\left[\left(\alpha_{i+1 / 2}^{n}\right)^{2} A_{i+1}^{n} A_{i}^{n} \sin \left(\theta_{i+1}^{n}-\theta_{i}^{n}\right)-\left(\alpha_{i-1 / 2}^{n}\right)^{2} A_{i}^{n} A_{i-1}^{n} \sin \left(\theta_{i}^{n}-\theta_{i-1}^{n}\right)\right]
\end{aligned}
$$

In contrast to the continuous case, it is unnecessary to neglect any small terms in the Lagrangian to obtain the identical formulation. Hayes's ensemble average over 
phase shift and Whitham's averaged Lagrangian give formally identical wave action conservation laws, without neglecting any higher order terms in the Lagrangian.

The variation of (30) with respect to $A_{i}^{n}$ gives a generalized numerical dispersion relation for variable coefficients:

$$
\begin{aligned}
0=\frac{1}{\Delta t^{2}} & {\left[A_{i}^{n}-\frac{1}{2} A_{i}^{n+1} \cos \left(\theta_{i}^{n+1}-\theta_{i}^{n}\right)-\frac{1}{2} A_{i}^{n-1} \cos \left(\theta_{i}^{n}-\theta_{i}^{n-1}\right)\right]+} \\
\frac{1}{\Delta x^{2}} & {\left[\frac{\left(\alpha_{i+1 / 2}^{n}\right)^{2}+\left(\alpha_{i-1 / 2}^{n}\right)^{2}}{2} A_{i}^{n}-\left(\alpha_{i+1 / 2}^{n}\right)^{2} \frac{1}{2} A_{i+1}^{n} \cos \left(\theta_{i+1}^{n}-\theta_{i}^{n}\right)\right.} \\
& \left.-\left(\alpha_{i-1 / 2}^{n}\right)^{2} \frac{1}{2} A_{i-1}^{n} \cos \left(\theta_{i}^{n}-\theta_{i-1}^{n}\right)\right]+\frac{\left(\beta_{i}^{n}\right)^{2}}{2} A_{i}^{n} .
\end{aligned}
$$

A discrete analog of the modulation equations (13)-(15) can be obtained by eliminating $\theta_{i}^{n}$ through the substitutions

$$
\theta_{i}^{n+1}-\theta_{i}^{n} \equiv-\omega_{i}^{n+1 / 2} \Delta t, \quad \theta_{i+1}^{n}-\theta_{i}^{n} \equiv \kappa_{i+1 / 2}^{n} \Delta x
$$

in (31) and (32). We then need the compatibility condition

$$
\frac{1}{\Delta t}\left(\kappa_{i+1 / 2}^{n+1}-\kappa_{i+1 / 2}^{n}\right)+\frac{1}{\Delta x}\left(\omega_{i+1}^{n+1 / 2}-\omega_{i}^{n+1 / 2}\right)=0 .
$$

Since the variables $A_{i}^{n}, \kappa_{i+1 / 2}^{n}$ and $\omega_{i}^{n+1 / 2}$ are all slowly varying, the discrete modulation equations could conceivably be solved on a coarser grid.

\subsection{Discrete multisymplectic integrators}

The wave action conservation law (31) may also be derived directly from a multisymplectic description. Consider a wave equation of the form (16).

Defining matrices $J^{+}, J^{-}, K^{+}, K^{-}$, to be the upper triangular and lower triangular parts of $J$ and $K$, respectively, the multisymplectic Euler discretization of (16) is given by (Moore \& Reich 2003a):

$$
\begin{aligned}
J^{+} & \frac{\boldsymbol{u}_{i}^{n+1}-\boldsymbol{u}_{i}^{n}}{\Delta t}+J^{-} \frac{\boldsymbol{u}_{i}^{n}-\boldsymbol{u}_{i}^{n-1}}{\Delta t} \\
& +K^{+} \frac{\boldsymbol{u}_{i+1}^{n}-\boldsymbol{u}_{i}^{n}}{\Delta x}+K^{-} \frac{\boldsymbol{u}_{i}^{n}-\boldsymbol{u}_{i-1}^{n}}{\Delta x}=\nabla S\left(\boldsymbol{u}_{i}^{n}, x_{i}, t_{n}\right) .
\end{aligned}
$$

Next assume a family of numerical solutions, smoothly and periodically dependent on the parameter $\theta_{0}$, and compute the vector inner product of (34) with $\partial_{\theta_{0}} \boldsymbol{u}_{i}^{n}$. Rearranging, and using the fact that $\left(J^{+}\right)^{T}=-J^{-},\left(K^{+}\right)^{T}=-K^{-}$gives the semidiscrete conservation law $\ddagger$

$$
\begin{aligned}
\partial_{\theta_{0}} & {\left[\frac{\left(\boldsymbol{u}_{i}^{n-1}\right)^{T} J^{+} \boldsymbol{u}_{i}^{n}-\left(\boldsymbol{u}_{i}^{n}\right)^{T} J^{+} \boldsymbol{u}_{i}^{n}}{\Delta t}+\frac{\left(\boldsymbol{u}_{i-1}^{n}\right)^{T} K^{+} \boldsymbol{u}_{i}^{n}-\left(\boldsymbol{u}_{i}^{n}\right)^{T} K^{+} \boldsymbol{u}_{i}^{n}}{\Delta x}-S\left(\boldsymbol{u}_{i}^{n}, x_{i}, t_{n}\right)\right] } \\
& +\frac{\left(\partial_{\theta_{0}} \boldsymbol{u}_{i}^{n}\right)^{T} J^{+} \boldsymbol{u}_{i}^{n+1}-\left(\partial_{\theta_{0}} \boldsymbol{u}_{i}^{n-1}\right)^{T} J^{+} \boldsymbol{u}_{i}^{n}}{\Delta t}+\frac{\left(\partial_{\theta_{0}} \boldsymbol{u}_{i}^{n}\right)^{T} K^{+} \boldsymbol{u}_{i+1}^{n}-\left(\partial_{\theta_{0}} \boldsymbol{u}_{i-1}^{n}\right)^{T} K^{+} \boldsymbol{u}_{i}^{n}}{\Delta x} \\
& =0 .
\end{aligned}
$$

Integrating around a closed loop in $\theta_{0}$, the first term disappears, leaving a discrete conservation law of the form (24), i.e.

$\ddagger$ This expression also suggests the form of semi-discrete energy-momentum conservation laws for semi-discretizations with the multisymplectic Euler scheme. 
Proposition 2 The multisymplectic Euler scheme (34) satisfies the discrete wave action conservation law (24) with

$\mathcal{A}_{i}^{n+1 / 2}=\oint\left(\partial_{\theta_{0}} \boldsymbol{u}_{i}^{n}\right)^{T} J^{+} \boldsymbol{u}_{i}^{n+1} d \theta_{0}, \quad \mathcal{B}_{i+1 / 2}^{n}=\oint\left(\partial_{\theta_{0}} \boldsymbol{u}_{i}^{n}\right)^{T} K^{+} \boldsymbol{u}_{i+1}^{n} d \theta_{0}$.

Since the above discretization is equivalent to the discrete Euler-Lagrange equations (23), these discrete density and flux functions are equal to (25). A larger class of multisymplectic discretizations will be dealt with in the next section.

\section{Wave action conservation for multisymplectic Runge-Kutta box schemes}

In this section we derive discrete wave action conservation laws for a popular class of multisymplectic methods, the Runge-Kutta box schemes (Reich 2000b). It is sufficient to consider a single space-time grid cell $\left[t_{0}, t_{1}\right] \times\left[x_{0}, x_{1}\right]$.

The discretization is a composition of an $s$-stage Runge-Kutta method in time and an $\tilde{s}$-stage method in space, and the method coefficients (Hairer et al. 1993) are denoted analogously

$$
\begin{aligned}
& c_{m}, b_{m}, a_{m \ell}, \quad m, \ell=1, \ldots, s \\
& \tilde{c}_{j}, \tilde{b}_{j}, \tilde{a}_{j k}, \quad j, k=1, \ldots, \tilde{s}
\end{aligned}
$$

The points $\left(\tau_{m}, \xi_{j}\right)$, where $\tau_{m}=t_{0}+c_{m} \Delta t$ and $\xi_{j}=x_{0}+\tilde{c}_{j} \Delta x$, are collocation points.

With these definitions, the Runge-Kutta box scheme semi-discretization is defined by a set of $s \times \tilde{s}$ collocation equations

$$
J \boldsymbol{T}_{j}^{m}+K \boldsymbol{X}_{j}^{m}=\nabla S\left(\boldsymbol{U}_{j}^{m}, \tau_{m}, \xi_{j}\right), \quad \begin{aligned}
& j=1, \ldots, \tilde{s}, \\
& m=1, \ldots, s,
\end{aligned}
$$

where $\boldsymbol{U}_{j}^{m}, \boldsymbol{T}_{j}^{m}$ and $\boldsymbol{X}_{j}^{m}$ are stage vectors approximating, respectively, $\boldsymbol{u}, \boldsymbol{u}_{t}$ and $\boldsymbol{u}_{x}$ at $\left(\tau_{m}, \xi_{j}\right)$. Additionally we have the relations

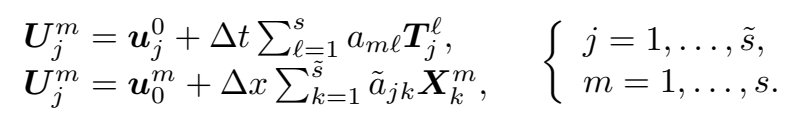

In (39) the quantities $\boldsymbol{u}_{j}^{0}$ and $\boldsymbol{u}_{0}^{m}$ approximate $\boldsymbol{u}$ on the cell faces at $\left(t_{0}, \xi_{j}\right)$ and $\left(\tau_{m}, x_{0}\right)$, respectively. The values on the opposite faces are denoted $\boldsymbol{u}_{j}^{1}$ and $\boldsymbol{u}_{1}^{m}$ and are obtained from

$$
\begin{aligned}
& \boldsymbol{u}_{j}^{1}=\boldsymbol{u}_{j}^{0}+\Delta t \sum_{m=1}^{s} b_{m} \boldsymbol{T}_{j}^{m}, \\
& \boldsymbol{u}_{1}^{m}=\boldsymbol{u}_{0}^{m}+\Delta x \sum_{j=1}^{m} \tilde{b}_{j} \boldsymbol{X}_{j}^{m},
\end{aligned}, \quad\left\{\begin{array}{l}
j=1, \ldots, \tilde{s} \\
m=1, \ldots, s .
\end{array}\right.
$$

Additional formulas are necessary to relate the above quantities to gridpoint values (Frank et al. 2006). However, the relations (38), (39) and (40) are sufficient to obtain the conclusions of this paper.

A Runge-Kutta box scheme is multisymplectic (i.e. satisfies a discrete local conservation law of symplecticity in the sense of (Bridges \& Reich 2001)) if both coefficient sets $\left\{c_{m}, b_{m}, a_{m \ell}\right\}$ and $\left\{\tilde{c}_{j}, \tilde{b}_{j}, \tilde{a}_{j k}\right\}$ define symplectic RK methods (Hairer et al. 2002), i.e.

$$
\begin{array}{ll}
b_{m} b_{\ell}-b_{\ell} a_{\ell m}-b_{m} a_{m \ell}=0, & \forall m, \ell, \\
\tilde{b}_{j} \tilde{b}_{k}-\tilde{b}_{k} \tilde{a}_{k j}-\tilde{b}_{j} \tilde{a}_{j k}=0, \quad \forall j, k .
\end{array}
$$

The following lemma is the discrete analog of (18). 
Lemma 1 Consider a skew-symmetric matrix $K \in \boldsymbol{R}^{N \times N}$ and a set of vectors $\boldsymbol{u}_{0}\left(\theta_{0}\right)$, $\boldsymbol{u}_{1}\left(\theta_{0}\right), \boldsymbol{U}_{j}\left(\theta_{0}\right), \boldsymbol{X}_{j}\left(\theta_{0}\right) \in \boldsymbol{R}^{N}, j=1, \ldots, \tilde{s}$, smoothly dependent on a parameter $\theta_{0}$ and satisfying the Runge-Kutta formulas

$$
\begin{aligned}
\boldsymbol{U}_{j} & =\boldsymbol{u}_{0}+\Delta k \sum_{k=1}^{\tilde{s}} \tilde{a}_{j k} \boldsymbol{X}_{k}, \quad m=1, \ldots, s \\
\boldsymbol{u}_{1} & =\boldsymbol{u}_{0}+\Delta k \sum_{j=1}^{\tilde{s}} \tilde{b}_{m} \boldsymbol{X}_{j} .
\end{aligned}
$$

For symplectic Runge-Kutta methods (41) the following identity holds:

$$
\sum_{j=1}^{\tilde{s}} \tilde{b}_{j} \partial_{\theta_{0}} \boldsymbol{U}_{j}^{T} K \boldsymbol{X}_{j}=\partial_{\theta_{0}}\left[\sum_{j=1}^{\tilde{s}} \tilde{b}_{j} \frac{1}{2} \boldsymbol{U}_{j}^{T} K \boldsymbol{X}_{j}\right]+\frac{1}{\Delta x}\left(F_{1}-F_{0}\right),
$$

with $F_{i}=\frac{1}{2} \partial_{\theta_{0}} \boldsymbol{u}_{i}^{T} K \boldsymbol{u}_{i}, i=0,1$.

Proof. Substitute (43) into the definition of $F^{1}$ to obtain

$$
\begin{gathered}
\partial_{\theta_{0}} \boldsymbol{u}_{1}^{T} K \boldsymbol{u}_{1}=\partial_{\theta_{0}} \boldsymbol{u}_{0}^{T} K \boldsymbol{u}_{0}+\Delta x \sum_{j=1}^{\tilde{s}} \tilde{b}_{j} \partial_{\theta_{0}} \boldsymbol{u}_{0}^{T} K \boldsymbol{X}_{j}+\Delta x \sum_{j=1}^{\tilde{s}} \tilde{b}_{j} \partial_{\theta_{0}} \boldsymbol{X}_{j}^{T} K \boldsymbol{u}_{0} \\
+\Delta x^{2} \sum_{j, k=1}^{\tilde{s}} \tilde{b}_{j} \tilde{b}_{k} \partial_{\theta_{0}} \boldsymbol{X}_{j}^{T} K \boldsymbol{X}_{k} .
\end{gathered}
$$

Solving (42) for $\boldsymbol{u}_{0}$, differentiating with respect to $\theta_{0}$, and substituting into the first series above yields

$$
\begin{aligned}
\sum_{j=1}^{\tilde{s}} \tilde{b}_{j} \partial_{\theta_{0}} \boldsymbol{u}_{0}^{T} K \boldsymbol{X}_{j} & =\sum_{j=1}^{\tilde{s}} \tilde{b}_{j} \partial_{\theta_{0}} \boldsymbol{U}_{j}^{T} K \boldsymbol{X}_{j}-\Delta x \sum_{j, k=1}^{\tilde{s}} \tilde{b}_{j} \tilde{a}_{j k} \partial_{\theta_{0}} \boldsymbol{X}_{k}^{T} K \boldsymbol{X}_{j} \\
& =\sum_{j=1}^{\tilde{s}} \tilde{b}_{j} \partial_{\theta_{0}} \boldsymbol{U}_{j}^{T} K \boldsymbol{X}_{j}-\Delta x \sum_{j, k=1}^{\tilde{s}} \tilde{b}_{k} \tilde{a}_{k j} \partial_{\theta_{0}} \boldsymbol{X}_{j}^{T} K \boldsymbol{X}_{k},
\end{aligned}
$$

where the skew-symmetry of $K$ been used. Similarly, the second series becomes

$$
\sum_{j=1}^{\tilde{s}} \tilde{b}_{j} \partial_{\theta_{0}} \boldsymbol{X}_{j}^{T} K \boldsymbol{u}_{0}=\sum_{j=1}^{\tilde{s}} \tilde{b}_{j} \partial_{\theta_{0}} \boldsymbol{X}_{j}^{T} K \boldsymbol{U}_{j}-\Delta x \sum_{j=1}^{\tilde{s}} \tilde{b}_{j} \tilde{a}_{j k} \partial_{\theta_{0}} \boldsymbol{X}_{j}^{T} K \boldsymbol{X}_{k} .
$$

Substituting the above two formulas into (45) gives

$$
\begin{gathered}
\partial_{\theta_{0}} \boldsymbol{u}_{1}^{T} K \boldsymbol{u}_{1}=\partial_{\theta_{0}} \boldsymbol{u}_{0}^{T} K \boldsymbol{u}_{0}+\Delta x \sum_{j=1}^{\tilde{s}} \tilde{b}_{j} \partial_{\theta_{0}} \boldsymbol{U}_{j}^{T} K \boldsymbol{X}_{j}+\Delta x \sum_{j=1}^{\tilde{s}} \tilde{b}_{j} \partial_{\theta_{0}} \boldsymbol{X}_{j}^{T} K \boldsymbol{U}_{j} \\
+\Delta x^{2} \sum_{j, k=1}^{\tilde{s}}\left(\tilde{b}_{j} \tilde{b}_{k}-\tilde{b}_{k} \tilde{a}_{k j}-\tilde{b}_{j} \tilde{a}_{j k}\right) \partial_{\theta_{0}} \boldsymbol{X}_{j}^{T} K \boldsymbol{X}_{k}
\end{gathered}
$$

For symplectic RK methods (41), the last term in (46) cancels. Finally we note that

$$
\partial_{\theta_{0}} \boldsymbol{U}_{j}^{T} K \boldsymbol{X}_{j}+\partial_{\theta_{0}} \boldsymbol{X}_{j}^{T} K \boldsymbol{U}_{j}=2 \partial_{\theta_{0}} \boldsymbol{U}_{j}^{T} K \boldsymbol{X}_{j}-\partial_{\theta_{0}}\left(\boldsymbol{U}_{j}^{T} K \boldsymbol{X}_{j}\right)
$$

and (44) follows. 
Next, by premultiplying (38) by $\partial_{\theta_{0}} \boldsymbol{U}_{j}^{m}$ and applying the quadrature over $j$ and $m$,

$$
\sum_{j, m} b_{m} \tilde{b}_{j}\left(\partial_{\theta_{0}} \boldsymbol{U}_{j}^{m}\right)^{T}\left[J \boldsymbol{T}_{j}^{m}+K \boldsymbol{X}_{j}^{m}-\nabla S\left(\boldsymbol{U}_{j}^{m}, \tau_{m}, \xi_{j}\right)\right]=0,
$$

and applying Lemma 1, we arrive at the semi-discrete conservation law

$$
\begin{aligned}
\partial_{\theta_{0}} \sum_{j, m} b_{m} \tilde{b}_{j} & {\left[\frac{1}{2}\left(\boldsymbol{U}_{j}^{m}\right)^{T} J \boldsymbol{T}_{j}^{m}+\frac{1}{2}\left(\boldsymbol{U}_{j}^{m}\right)^{T} K \boldsymbol{X}_{j}^{m}-S\left(\boldsymbol{U}_{j}^{m}, \tau_{m}, \xi_{j}\right)\right] } \\
+ & \frac{1}{\Delta t}\left(A^{1}-A^{0}\right)+\frac{1}{\Delta x}\left(B_{1}-B_{0}\right)=0
\end{aligned}
$$

where

$$
\begin{array}{rlrl}
A^{n} & =\sum_{j=1}^{\tilde{s}} \tilde{b}_{j} \frac{1}{2}\left(\partial_{\theta_{0}} \boldsymbol{u}_{j}^{n}\right)^{T} J \boldsymbol{u}_{j}^{n}, & n=0,1, \\
B_{i}=\sum_{m=1}^{s} b_{m} \frac{1}{2}\left(\partial_{\theta_{0}} \boldsymbol{u}_{i}^{m}\right)^{T} K \boldsymbol{u}_{i}^{m} & i=0,1 .
\end{array}
$$

Finally, taking the ensemble average of (47) around a loop in $\theta_{0}$ proves

Proposition 3 The multisymplectic Runge-Kutta discretization (38)-(41) satisfies the following discrete conservation law of wave action

$$
\frac{1}{\Delta t}\left(\mathcal{A}^{1}-\mathcal{A}^{0}\right)+\frac{1}{\Delta x}\left(\mathcal{B}_{1}-\mathcal{B}_{0}\right)=0
$$

where

$$
\begin{array}{ll}
\mathcal{A}^{n}=\frac{1}{2 \pi} \oint \sum_{j=1}^{\tilde{s}} \tilde{b}_{j} \frac{1}{2}\left(\partial_{\theta_{0}} \boldsymbol{u}_{j}^{n}\right)^{T} J \boldsymbol{u}_{j}^{n} d \theta_{0}, & n=0,1, \\
\mathcal{B}_{i}=\frac{1}{2 \pi} \oint \sum_{m=1}^{s} b_{m} \frac{1}{2}\left(\partial_{\theta_{0}} \boldsymbol{u}_{i}^{m}\right)^{T} K \boldsymbol{u}_{i}^{m} d \theta_{0}, & i=0,1 .
\end{array}
$$

Note that the discrete wave action conservation law (48) holds for nonlinear problems, for problems (16) where $S$ depends explicitly on the space-time coordinated $x$ and $t$ (where energy and momentum are not conserved), and for any tensor product grid (we have looked at a single grid cell here, without any reference to the size of neighboring cells). This discrete conservation law is the discrete analog of the general wave action conservation law of (Hayes 1970) and is an exact law. However, like the result of (Hayes 1970), the utility of this result depends on the identification of the ensemble parameter $\theta_{0}$.

Remark. By identifying $\theta_{0}$ with another coordinate direction, say $y$, which need not be periodic, the semi-discrete conservation law (47) corresponds to the conservation of momentum associated with translation symmetry in the $y$ dimension (since $S$ exhibits no explicit dependence on $y$ ). As such, (47) is a more general statement of a semi-discrete conservation law of semi-discretizations, with respect to the momentum in the nondiscretized directions (Frank 2006). This semi-discrete conservation law has been noted in other contexts before, see (Reich $2000 \mathrm{~b}$, Reich $2000 a$, Bridges \& Reich 2001, Moore \& Reich 2003a, Moore \& Reich 2003b, Hong \& Li 2006). 


\section{Numerical experiment}

In this section we illustrate discrete wave action conservation using the discrete variational/multisymplectic Euler method (23),(34), applied to a slowly modulated wave train solution of the Klein-Gordon equation (4). The domain is the interval $x \in[0, L)$ with $L=2 \pi / \varepsilon$ and periodic boundary conditions. The coefficients $\alpha$ and $\beta$ are chosen to be

$\alpha=1+\frac{1}{5} \sin \left(\frac{\pi}{27} \varepsilon t\right) \exp \left[-25\left(\frac{x}{L}-\frac{1}{2}\right)^{2}\right], \quad \beta=1-\cos \left(\frac{\pi}{20} \varepsilon t\right) \exp \left[-25\left(\frac{x}{L}-\frac{1}{2}\right)^{2}\right]$.

We take as initial condition a uniform, right-traveling wave train

$u(0, x)=\sin \left(\kappa x+\theta_{0}\right), \quad v(0, x)=u_{t}(0, x)=-\omega \cos \left(\kappa x+\theta_{0}\right), \quad \kappa=\omega=\frac{4 \pi}{\varepsilon L}$.

For the discretization, we have $N=30 / \varepsilon, \Delta t=\varepsilon L / N$, and integrate to time $160 / \varepsilon$.

For a linear problem, it is possible, by taking the derivative of (34) with respect to $\theta_{0}$, to also integrate numerically and determine $u_{\theta_{0}}$ and $v_{\theta_{0}}$, using initial conditions

$$
u_{\theta_{0}}(0, x)=\cos \left(\kappa x+\theta_{0}\right), \quad v_{\theta_{0}}(0, x)=\omega \sin \left(\kappa x+\theta_{0}\right) .
$$

Then, under the assumption that $u_{i}^{n}=\tilde{A}_{i}^{n} \sin \left(\tilde{\theta}_{i}^{n}+\theta_{0}\right), \partial_{\theta_{0}} u_{i}^{n}=\tilde{A}_{i}^{n} \cos \left(\tilde{\theta}_{i}^{n}+\theta_{0}\right)$, we can approximate the amplitude and phase of the numerical solution by

$$
\tilde{A}_{i}^{n}=\sqrt{\left(u_{i}^{n}\right)^{2}+\left(\partial_{\theta_{0}} u_{i}^{n}\right)^{2}}, \quad \tilde{\theta}_{i}^{n}=\tan ^{-1} \frac{u_{i}^{n}}{\partial_{\theta_{0}} u_{i}^{n}} .
$$

Alternatively, we can integrate the discrete modulation equations (31), (32) to approximate $A_{i}^{n}$ and $\theta_{i}^{n}$. A comparison of the numerical solution and the amplitude obtained by a separate integration of (31), (32) is shown in Figure 1.

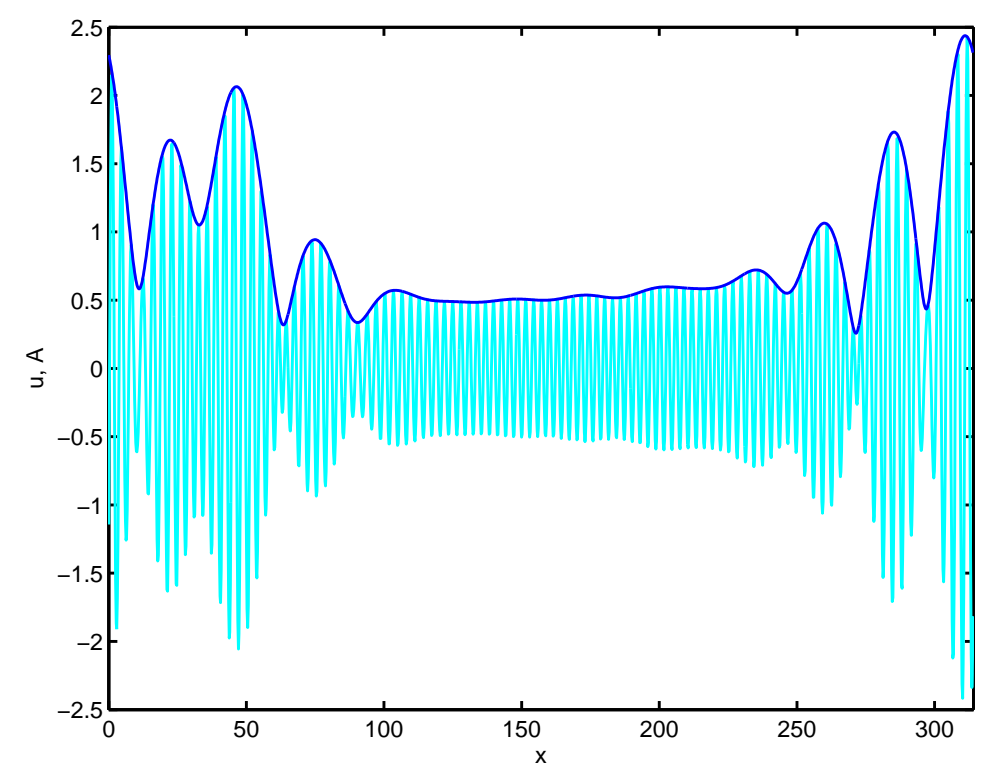

Figure 1. Solution $u_{i}^{n}$ (light blue) and amplitude $A_{i}^{n}$ (blue) at time $T=160 / \varepsilon$ for $\varepsilon=0.02$. 
The wave action density is given by (28). The total wave action at time $t_{n}$ is given by

$$
\mathcal{A}^{n}=\sum_{i} \mathcal{A}_{i}^{n} \Delta x
$$

This quantity is conserved to machine precision, as can be seen by applying the summation above to (31) with periodic boundary conditions. If we substitute $\tilde{A}_{i}^{n}$ and $\tilde{\theta}_{i}^{n}$ as determined from (49) into (31), the residual is nonzero. Nonetheless, the total wave action is conserved in this case as well. Figure 2 compares the relative variation in total wave action to that of total energy for $\varepsilon=0.05$. The total wave action is $2 \pi / \varepsilon$ and the initial energy is $13.3 / \varepsilon$.

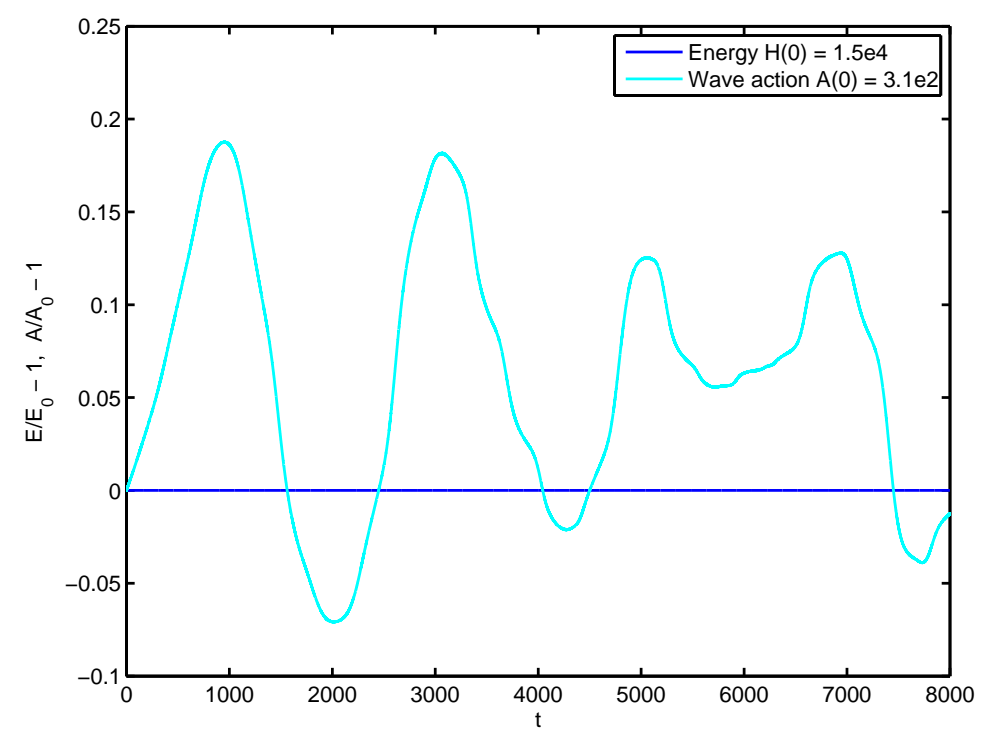

Figure 2. Relative variation in total energy (light blue) and total wave action (blue) as a percentage of the initial value. $\varepsilon=0.02$.

As a measure of the accuracy of the discrete modulation equations, we can measure the approximation error in the $L_{\infty}$-norm

$$
e^{n}=\max _{i}\left|u_{i}^{n}-A_{i}^{n} \sin \theta_{i}^{n}\right|,
$$

where again $A_{i}^{n}$ and $\theta_{i}^{n}$ are obtained from a separate integration of (31), (32). This quantity is plotted as a function of time in Figure 3 for $\varepsilon=0.4,0.2,0.1$ and 0.05 . The results are scaled in time to fit on one plot. The similarity of the error evolution suggests that the discrete modulation equations approximate the numerical solution to order $\mathcal{O}(\varepsilon)$ for intervals of $\mathcal{O}\left(\varepsilon^{-1}\right)$.

\section{Concluding remarks}

In this paper we have derived discrete wave action conservation laws for the discrete variational/multisymplectic Euler method and the class of multisymplectic RungeKutta box schemes. Within the variational framework it is also possible to derive 


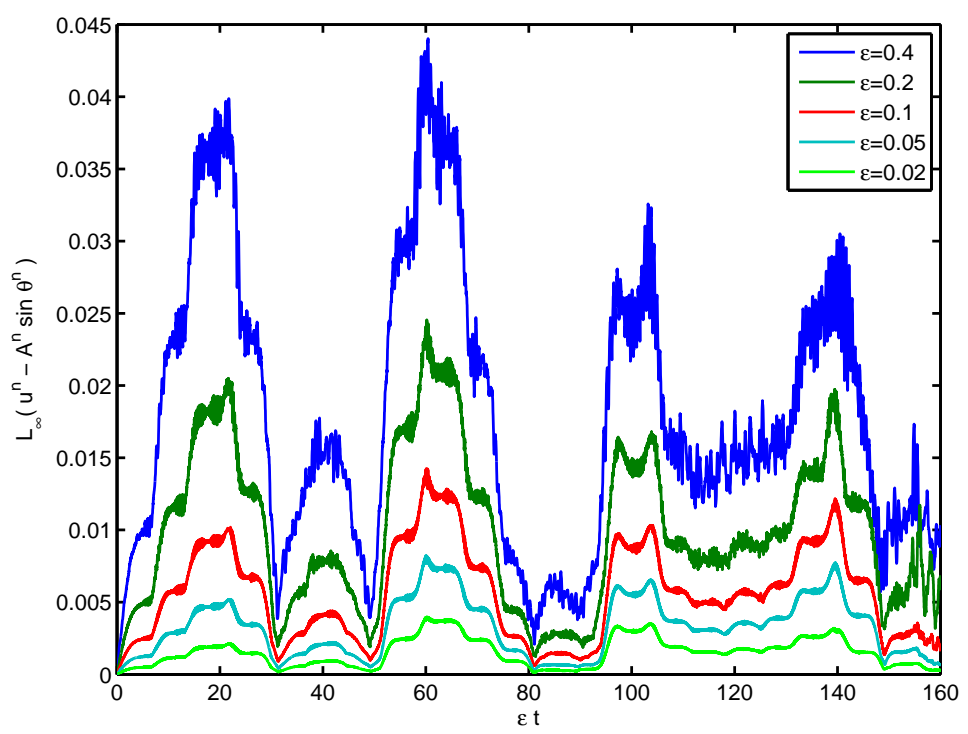

Figure 3. Approximation of the solution by the discrete modulation equations as a function of $\varepsilon$.

discrete modulation equations. Numerical experiments confirm that wave action is conserved to machine precision and also suggest that the discrete modulation equations approximate the numerical solution to order $\mathcal{O}(\varepsilon)$ for intervals of $\mathcal{O}\left(\varepsilon^{-1}\right)$. Since the discrete modulation equations can be written in terms of slowly varying amplitude, wavenumber and frequency, it might be feasible to solve these on a coarse mesh.

Because the discrete variational framework also gives access to the full modulation equations, a general development of wave action for this class would be desirable.

The wave action conservation laws of this paper apply more generally to discretized nonlinear PDEs, and in the case of the box schemes, also to nonuniform space-time grids. To actually compute the wave action, however, one must have an explicit expression for a periodic wave train, just as in the continuous case.

Wave action is the generalization to PDEs of the concept of an adiabatic invariant. The classical example of an adiabatic invariant is the slowly modulated harmonic oscillator, obtained from (4) by setting $\alpha \equiv 0$ (Arnold 1989). Reich has shown that symplectic integrators conserve adiabatic invariants over exponentially long times (Reich 1999, Cotter \& Reich 2004), see also (Cotter 2004). Estimates of the longevity of wave action conservation for PDE discretizations are currently lacking.

\section{Acknowledgments}

I thank Tom Bridges for comments on the manuscript and the anonymous referees for critical remarks that led to a number of improvements. Funding through a Veni grant from the Netherlands Organization for Scientific Research is also gratefully acknowledged. 


\section{References}

Andrews D G \& McIntyre M E 1978 J. Fluid. Mech. 89, 647-664.

Arnold V I 1989 Mathematical Methods of Classical Mechanics Springer, New York.

Bretherton F P \& Garrett C J R 1969 Proc. Roy. Soc. A. 302, 529-554.

Bridges T J 1997a Proc. R. Soc. Lond. A 453, 1365-1395.

Bridges T J 1997b Math. Proc. Camb. Phil. Soc. 121, 147-190.

Bridges T J \& Reich S 2001 Phys. Lett. A 284, 184-193.

Cotter C J 2004 Model Reduction for Shallow Water Dynamics: Balance, Adiabatic Invariance and Subgrid Modelling PhD thesis Imperial College of London.

Cotter C J \& Reich S 2004 BIT 44, 439-455.

Frank J 2006 A note on semi-discrete conservation laws and conservation of wave action by multisymplectic runge-kutta box schemes Technical Report MAS-E0603 CWI, Amsterdam.

Frank J, Moore B \& Reich S 2006 SIAM J. Sci. Comput. . to appear.

Grimshaw R 1984 Ann. Rev. Fluid Mech. 16, 11-44.

Hairer E, Lubich C \& Wanner G 2002 Geometric Numerical Integration: Structure Preserving Methods for Ordinary Differential Equations Springer, Berlin.

Hairer E, Nørsett S \& Wanner G 1993 Solving Ordinary Differential Equations I: Nonstiff Problems 2nd edn Springer-Verlag, Berlin.

Hayes W D 1970 Proc. R. Soc. Lond. A 320, 187-208.

Hong J \& Li C 2006 J. Comput. Phys. 211, 448-472.

Marsden J E, Patrick G W \& Shkoller S 1998 Comm. Math. Phys. 199, 351-395.

Marsden J E \& Ratiu T S 1994 Introduction to Mechanics and Symmetry Springer-Verlag, New York.

Marsden J E \& West M 2001 Acta Numerica pp. 357-514.

Moore B \& Reich S 2003a Numer. Math. 95, 625-652.

Moore B \& Reich S 2003 b Future Generation Computer Systems 19, 395-402.

Reich S 1999 Appl. Numer. Math. 29, 45-56.

Reich S 2000a BIT 40, 559-582.

Reich S $2000 b$ J. Comput. Phys. 157, 473-499.

Whitham G B 1965 J. Fluid. Mech. 22, 273-283.

Whitham G B 1970 J. Fluid Mech. 44, 373-395.

Whitham G B 1999 Linear and Nonlinear Waves Wiley, New York. 